M. Malinvaud se rappelle avoir vu assez fréquemment diverses Fougères, Polypodium, Asplenium, etc., végétant sur des trones d'arbres; mais, son attention n'étant pas appelée sur l'intérêt de ces observations, il n'en a pas noté les circonstances qui permettraient de faire apprécier la nature du phénomène.

MM. F. Camus, Lutz et Zeiller ont fait des remarques analogues.

M. Malinvaud donne lecture de la communication suivante :

\title{
LE PIN a CROChETS SPONTANÉ dANS LES CÉVENNES;
}

\section{par H. A. I'ALVERNY.}

Les Flores et Catalogues n'ont signalé jusqu'ici la présence d'aucune des formes de Pinus montana Duroi, dans le Plateau Central et les Cévennes.

Il en existe cependant au moins un massif spontané dans les monts du Forez, sur la commune de Chalmazel, près Boën (Loire).

Isolé dans un vaste désert de bruyère, à 1350 mètres d'altitude, sur roches granitiques, dans le bassin de réception du ruisseau torrentiel de Cluzel, - l'une des branches du Lignon, - est un petit bois communal de 12 hectares environ. La moitié supérieure, sur un versant à pente assez raide au sud-est, est peuplée de Sapin et de Hètre; on trouve le Pin à crochets pur au-dessous, où commence une tourbière très étendue, au parcours pénible et dangereux.

Ce Pin à crochets est le Pinus montana Duroi var. uncinata Ram. subvar. elala F. Gérard (in Magnier). Il est identique à la plante assez répandue dans les tourbières du Jura (Fresne, etc.) et des Vosges (Gérardmer, etc.) (1).

Les arbres sont bien venants, atteignent 6 ou 7 mètres de hauteur et se régénèrent parfaitement. Ils forment un massif clair, puis des bouquets semés sur la lisière et aux bords du ruisseau. Ce massif est désigné par les habitants sons le nom de La Pigne,

(1) Cf. Exsicc. Soc. Rochel., $\mathrm{n}^{\circ} 4652$, leg. F. Gérard, au Beillard, Gérardmer. 
D'Alverny. - LE PIN A Grochets DANS LEj CÉvennes.

dont on reconnait aisément l'étymologie (Pinea). Or on ne nomme jamais ainsi les peuplements de Pin silvestre, nombreux dans la région. L'autre ${ }_{\mathbf{6}}$ Pin avait donc été remarqué : singulier en effet, à une altitude où tous les bois sont de Sapin et Hètre, et où le Pin silvestre ne vit plus.

Dans ces pâturages, dont le déboisement a fait un désert pelé ou couvert de bruyère, la permanence de ce massif, d'ailleurs peu dégradé, est remarquable. L’éloignement de toute habitation, même des jasseries (chalets-fromageries), l'a préservé du pillage des hommes ; le danger de la tourbière a empèché aussi, dans une certaine mesure, les dégàls des troupeaux.

Le Pin à crochets, employé avec grand succès dans les reboisements de la Lozère et du Gard, était donc aussi spontané dans les Cévennes. Il faudra voir là une précieuse indication forestière, et c'est à ce titre que nous avons un peu insisté sur cette petite découverte.

Mais ce fait éveille aussi des remarques intẻressantes en botanique pure.

Le Pinus montana Duroi (1771), nettement caractérisé dans sa forme typique et très distinct des autres espèces du genre, par son port, son habitat et sa distribution géographique aussi bien que par ses caractères morphologiques, présente au moins trois formes importantes, jadis considérées comme espèces. Rattachées avec raison au type de l'espèce de Duroi, ces variétés n'en ont pas moins un grand intérèt botanique.

Le $P$. montana var. uncinata ( $P$. uncinala Ramond) est la seule Conifère subalpine spontanée aux Pyrénées; on ne peut, en effet, considérer comme telle le $P$. silvestris qui s'élève parfois dans les forèts subalpines, mais qui descend surtout vers les zones inférieures des montagnes, jusqu'à 600 mètres et au-dessous. Le Pin à crochets, comme le nomment les forestiers, forme toutes les forèts supérieures de la Cerdagne et du Capsir (jusque vers 2300 mètres), des Pyrénées catalanes et aragonaises (Laguna).

Il est aussi la seule essence subalpine du Ventoux, promontoire des Alpes au voisinag̣e dưr Rhòne.

Dans le massif des Alpes occidentales, il est partout subordonné au Mélèze ou à l'Épicea, suivant les cas, formant un peu partout des bois, de peu d'étenduejen général.

Assez répandu, mais toujours en mélange dans les Alpes cenT. XLIX.

(SÉANCES) 5 
trales (Christ), les Carpathes et les Sudètes (Pax), il est rare dans les Apennins et les montagnes de Macédoine (Boissier), et sporadique au Claucase (Radde). Cette forme paraît tout à fait indépendante de la nature du sol.

Une forme (var. elata F. Gérard) plus maigre, plus élancée, mais à peine séparable du type, couvre d'une forèt continue la grande tourbière à l'aval du lac de Gérardmer et plusieurs autres tourbières des Vosges françaises.

C'est avec raison, sans doute, que M. E. Krause (1) nie l'existence, dans les Vosges, de la variété Mughus; il eût été plus complet s'il avait précisé le fait de l'existence spontanée du Pin de montagne dans ce massif, sous une forme rapprochée du type (Pin à crochets).

Vers la limite supérieure, dans cette zone contestée dont M. A. Mathey a si bien écrit l'histoire (2), en tout cas au delà de $2000 \mathrm{~m}$. dans nos montagnes de France, il arrive que des individus du type (Pin à crochets) sont écrasés ou brisẻs par la neige el qu'ils se reconstituent en prenant une forme buissonneuse; il arrive aussi qu'ils soient abroutis par le bétail. Il faut se garder de les confondre avec les variétés suivantes caractérisées par les détails de leurs cônes mieux encore que par leur port.

Le Pinus montana var. Pumilio (P. Pumilio Hrenke, 1791) est ordinairement un arbrisseau à branches couchées et tortueuses; plus répandu que le type dans les massifs montagneux de l'Europe centrale et orientale, à ce qu'il semble, il ne parait pas exister du tout en France.

Le Pinus montana var. Mughus (P. Mughus Scopoli, 1772) est aussi un arbrisseau trainant. Il paraît fréquent dans les Alpes orientales, et n'existe pas en France, où les anciens botanistes l'ont sans doute confondu avec une des formes que nous rattachons ici au type (Pin à crochets).

Un examen superficiel peut assurément laisser appliquer la diagnose de la variété Pumilio à un individu du type français uncinata déformé aux hautes stations. Koch (Consp. Fl. Germ.) encourage encore des confusions regrettables en nommant le Pumilio: P. Mughus var. $\beta$. Pumilio. Nais ceux-là ne sauraient s'y tromper

(1) E.-H.-L. Krause, Ueber das angebliche Indigenat der Pinus Mughus in den Vogesen (Ber. d. deütsch. bot. Ges. XII, 1894, pp. 236-239).

(2) A. Mathey, Le paturage en montagne. Besançon, 1900, pp. 94 et suiv. 
qui ont vu le vrai $P$. Mughus Scop. dans sa station : cette grande broussaille traìnante est trop commune et trop caractéristique de l'extrême zone subalpine dans les grándes Alpes orientales, au Tyrol, en Carinthie, etc. D'ailleurs elle semble franchement calcicole, et le Pin à crochets, commeil a été noté plus haut, ne marque aucune préférence relative à la composition du sol.

L'existence d'un bois de Pin à crochets dans le Massif central a un autre intérêt. Les phytogéographes contemporains se préoccupent de la distribution des Conilères aux époques géologiques antérieures à la nôlre. M. Fliche a signalé des faits importants relatifs à la distribution du Pin silvestre, du Mélèze et de l'Épicea pendant la période de la grande extension des glaciers.

Nous savons, gràce à lui, que le Pin de montagne habitait alors le plateau lorrain (Jarville, près de Nancy, Bois-l'Abbé, près d'Épinal), que le Pin silvestre était spontané dans le bassin de la Seine pendant la période de réchauffement interglaciaire (1). Nous savons encore que le Pin Laricio existait à l'époque quaternaire aux environs de Montpellier (2).

N'y a-t-il pas lieu de considérer le bois de Pin à crochets de Chalmazel, comme un témoin, comme un survivant d'une époque antérieure, au même titre qu'on voit des témoins de l'époque quaternaire dans les quatre îlots de Pin Laricio qui, sous une forme spéciale (Pinus Laricio var. Salzmanni), représentent actuellement l'espèce à l'état spontané en France.(3)?

\section{Guérin fait la communication suivante :}

(1) P. Fliche, Bull. Soc. géolog. de France, $3^{e}$ série, XXV, 1897, p. 95?,

(2) G. Planchon, Étude des Tufs de Montpellier. Paris et Montpellier. 1864.

(3) G. Fabre, Compt. rend. Acad. Sc. Paris, 13 janvier 1896. 


\section{$2 \mathrm{BHL}$ Biodiversity Heritage Library}

Alverny, André d'. 1902. "Le Pin A Crochets Spontané Dans Les Cévennes." Bulletin de la Société botanique de France 49, 64-67. https://doi.org/10.1080/00378941.1902.10828957.

View This Item Online: https://www.biodiversitylibrary.org/item/8671

DOI: https://doi.org/10.1080/00378941.1902.10828957

Permalink: https://www.biodiversitylibrary.org/partpdf/160239

\section{Holding Institution}

Missouri Botanical Garden, Peter H. Raven Library

\section{Sponsored by}

Missouri Botanical Garden

\section{Copyright \& Reuse}

Copyright Status: Public domain. The BHL considers that this work is no longer under copyright protection.

This document was created from content at the Biodiversity Heritage Library, the world's largest open access digital library for biodiversity literature and archives. Visit BHL at https://www.biodiversitylibrary.org. 